\title{
Geographic variation and determinants of help seeking behaviour among married women subjected to intimate partner violence: evidence from national population survey
}

Muluken Dessalegn Muluneh ${ }^{1,2^{*}}$, Yeshemebet Worku Alemu ${ }^{3}$ and Maereg Wagnew Meazaw ${ }^{4}$

\begin{abstract}
Background: Help seeking behaviour amongst married women who experienced Intimate Partner Violence (IPV) has received limited attention in Africa. This study examines the geographic variation and investigates determinants of help seeking behaviour amongst married women in Ethiopia.

Methods: This study analysed data from the 2016 Ethiopian Demographic and Health Survey (EDHS). Data was extracted for married women age 15-49 years old who experienced IPV. Factors associated with help seeking behaviour were identified using multiple logistic regression adjusted for clustering and weighing. The weighted proportion of factors associated with help seeking behaviour was exported to ArcGIS to conduct autocorrelation analysis.
\end{abstract}

Results: The prevalence of help seeking behaviour among married women who experienced IPV was 19.8\% (95\% Cl: 15.9-24.3\%). Only 9.2\% of them sought help from a formal source (such as police, lawyer or doctor). Multiple logistic regression analyses showed physical violence (Adjusted odds ratio $(A O R)=2.76)$, educational attainment $(A O R=2.1)$, a partner's alcohol consumption ( $A O R=1.9$ ), partner's controlling behaviour ( $A O R=2.4)$, partner's employment status, $(A O R=1.9)$ and wealth index $(A O R=2.8)$ were significantly associated factors with help seeking behaviour among married women who experienced IPV in Ethiopia $(P<0.05)$. Women in Benishangul-Gumuz, Gambella, Harari, Western and Eastern Amhara, and Afar had the lowest odds of help seeking behaviour $(P<0.001)$ after experiencing IPV.

(Continued on next page)

\footnotetext{
* Correspondence: destamule@gmail.com

${ }^{1}$ Monitoring Evaluation and Research Department, Amref Health Africa in Ethiopia, Addis Ababa, Ethiopia

${ }^{2}$ School of Nursing and Midwifery, Western Sydney University, Sydney, Australia

Full list of author information is available at the end of the article
}

C C The Author(s). 2021 Open Access This article is licensed under a Creative Commons Attribution 4.0 International License, which permits use, sharing, adaptation, distribution and reproduction in any medium or format, as long as you give appropriate credit to the original author(s) and the source, provide a link to the Creative Commons licence, and indicate if changes were made. The images or other third party material in this article are included in the article's Creative Commons licence, unless indicated otherwise in a credit line to the material. If material is not included in the article's Creative Commons licence and your intended use is not permitted by statutory regulation or exceeds the permitted use, you will need to obtain permission directly from the copyright holder. To view a copy of this licence, visit http://creativecommons.org/licenses/by/4.0/. The Creative Commons Public Domain Dedication waiver (http://creativecommons.org/publicdomain/zero/1.0/) applies to the data made available in this article, unless otherwise stated in a credit line to the data. 


\begin{abstract}
(Continued from previous page)
Conclusion: The findings of this study suggest that poor help seeking behaviour for married women experiencing IPV is a significant public health problem in Ethiopia. Multiple interrelated factors were associated with poor help seeking behaviour. These factors include women's level of educational attainment, women experiencing physical violence, partners exhibiting controlling behaviour, partner's alcohol consumption, the employment status of the partner, and wealth status of the household were important predictors of help seeking behaviour. Policies and interventions need to be tailored to address these factors to improve women's health outcomes and to prevent IPV.
\end{abstract}

Keywords: Intimate partner violence, Married women, Geographic, Hierarchical logistic, DHS, Ethiopia

\section{Introduction}

The World Health Organization (WHO) defines intimate partner violence (IPV), as "any behaviour within an intimate relationship that causes physical, psychological or sexual harm to those in the relationship that includes acts of physical aggression, psychological abuse, sexual coercion, and controlling behaviours" [1]. Globally, 30\% of women experience gender based violence (GBV) [2]. In Sub-Saharan Africa, the prevalence of GBV exceeds the global rate at $44 \%$ prevalence [3]. IPV is the most common form of GBV, accounting for 75 to $85 \%$ of violence against women globally $[4,5]$. IPV has been declared a universal problem across all contexts and countries, but distribution of occurrence widely varies [5-7]. A recent nationwide survey in Ethiopia revealed the lifetime prevalence of any form of IPV (physical, emotional or sexual) was $34 \%$ [8]. More broadly, $57 \%$ of married women reported experiencing at least one form of controlling behaviour from their partner [8].

In addition to the high prevalence of IPV globally, the prevalence of help seeking behaviour reported by women who have experienced IPV is low $[9,10]$. The WHO's multi-country study of IPV reported that $55-95 \%$ of women who had experienced physical or sexual IPV have never sought help from a formal institution [5, 11, 12]. The literature suggests women who have experienced IPV rarely report their experiences to formal sources and many do not seek any form of support [10]. The prevalence of help-seeking and disclosure behaviour, however, varies widely between countries $[5,11,13,14]$.

Studies show a clear pattern in which gender equity in a society influences the help seeking behaviour of women. Women in low-income countries with large gender inequality and rigid gender roles seek less help than women in countries with higher levels of gender equality, where gender roles are fluid $[15,16]$. In Ethiopia and other SSA countries, rigid gender roles are widespread. Patriarchal values that emphasize male authority in several aspects of everyday life are deep-rooted. This patriarchal culture enforces male dominance and enables controlling and violent behavior [9, 16-18].

Although Ethiopia has set various legal declarations and policies that support gender equality, rigid gender roles and inequity remains rampant. These governmental declarations include the 1994 Constitution that promotes equality of men and women [19]; the 2000 Revised Family Law on marriage and resource sharing [20], and the 2004 Penal Code of Ethiopia to combat violence against women [21]. In addition, Ethiopia has signed international and continental agreements and criminal codes that address acts of violence against women. In spite the legal measures and policy enactment, IPV is still widespread and help seeking behaviour by women in Ethiopia remains poor. A large majority of existing programs and policies target survivors who have already accessed services. There is limited research focused on the help seeking behaviour of IPV survivors in developing countries, including Ethiopia. To better understand the hidden nature of violence and to better reach women experiencing IPV, it is critical the rate and patterns of reporting, and the determinants of help seeking behaviour are explored. Therefore, this study aims to conduct geospatial analysis and to investigate associated factors of help seeking behaviour among married women of reproductive age between 15 to 49 years old in Ethiopia.

\section{Methods}

\section{Study setting, design and data source}

Ethiopia is the second most populous country in Africa. Located in Eastern SSA [22]. Ethiopia has one of the lowest gender equality performance indicators in SSA countries [22]. Women and girls in Ethiopia are strongly disadvantaged in the areas of literacy, health, livelihood, and basic human rights [22]. They have the lowest socioeconomic status among the population and lack adequate social support networks [23-26].

This study uses data from the 2016 Ethiopian Demographic and Health Survey (EDHS). The 2016 EDHS survey is the fourth population cross sectional survey to be conducted by the Ethiopian Central Statistical Agency (CSA). The survey was conducted in nine administrative regional states, Tigray, Afar, Amhara, Oromia, Somali, Benishangul-Gumuz, Southern Nation and Nationalities, Harari and Gambella, and two administrative cities (Addis Ababa and Dire Dawa). During the survey collection 
period, all individuals who spent the night in that particular area were interviewed (defacto data collection method) during door to door survey [23]. The 2016 EDHS was the first ever to include a module of questions related to domestic violence. According to the ethical guidelines of the WHO, single women were randomly selected from each household to complete the survey [27].

\section{Data extraction and instrument}

The EDHS employed standard questionnaires developed by the MEASURE DHS program. The survey datasets were downloaded from the MEASURE DHS website [28] in STATA format with permission. Since the domestic violence data was not collected in the EDHS 2000, EDHS 2005 and EDHS 2011, only 2016 EDHS data was considered for this study. After reviewing the detailed datasets and coding, further data recoding took place. All potential factors including individual, relationship, community, and social determinants were extracted from the datasets.

\section{Sample size and sampling procedure}

Sampling for this study was based on standard EDHS sampling techniques. The 2016 EDHS used 84,915 enumeration areas; each enumeration area has an average of 181 households, from nine regions and two city administrations. Then, two stage stratified cluster sampling was used. First, 645 enumeration areas were selected from urban (202 enumeration areas) and rural (443 enumeration areas) areas based on proportional size allocation. In the second stage, on average, 28 households were selected per enumeration area using systematic random sampling. For the violence against women module, only one woman per household was interviewed. Overall, 5860 women were interviewed about their experiences of GBV with a response rate of $97 \%$ [23]. A total of 4720 married women responded to the spousal violence questionnaire. Only married women who had an experience of violence $(n=1423)$ were included in this study to assess their help seeking behaviour.

\section{Variables}

This study assesses help seeking behaviour (Yes/No) as the dependent variable. This help seeking behaviour was examined against four levels of independent variables including individual variables (women characteristics), partner/ family characteristics, community level, and societal level variables. A literature review conducted on associated factors in SSA and the EDHS datasets were used to identify and select the variable categories presented in Table 1.

\section{Analysis plan}

Both descriptive and multivariable logistic regression analysis was conducted. As recommended by Hosmer and Lemeshow, statistically significant variables at $p$-value $<$ 0.25 during bivariate logistic regression analysis were used for the multivariable logistic regression analysis [29]. A staged multivariable logistic regression adjusted for clustering and sampling weights was conducted. The multivariate analysis was conducted to identify the independent effect of the various independent variables on help seeking behaviour after controlling for other factors that include individual, partner, community, and societal factors (Table 2).

The odds ratios (ORs) and 95\% confidence intervals (CIs) were calculated to assess the adjusted risk factors that affect the study's outcome. Those with $p<0.05$ were retained in the final model.

\section{Spatial analysis}

Help seeking behaviour data was exported to ArcGIS 10.3.4 to visualize estimations. Geospatial analysis was conducted to determine whether the help seeking behaviour was significantly clustered or randomly clustered in particular locations of Ethiopia. Spatial autocorrelation in the prevalence of help seeking behaviour was measured using the Global Moran's I statistic [30]. The Global Moran's I tool was used to determine the overall pattern and trend of the data. The geographic variation of clustered IPV help seeking behaviour prevalence was assessed using $\mathrm{Z}$-score values and $p$-values. A positive $\mathrm{Z}$ score indicates high help seeking behaviour prevalence clustering and a negative $\mathrm{Z}$ score indicates low clustering. A positive Moran's I index value indicates a tendency toward clustering, while a negative Moran's I index value indicates a tendency toward dispersion [31].

A hot spot analysis was then conducted utilizing the Hot Spot Analysis (Getis-Ord Gi*) tool in ArcGIS [32]. Hot spot analyses display high or low clusters of help seeking behaviours. The hot spot analysis image conveyed that there are areas within Ethiopia with high spatial clustering or better IPV help seeking behaviour than would be assumed by pure randomness.

\section{Results}

The graph below shows the experience of IPV among married women age 15-49 years old was $34 \%$. Both physical and emotional violence were the most reported forms of violence. Among women who had experienced IPV, 20\% sought help from a social service provider (Fig. 1). The majority of women (80\%) never sought help or told anyone about the physical, sexual or emotional violence they encountered from their partner.

Below graph (Fig. 2) shows that among a small proportion of women who sought help, the top most 
Table 1 Variables for identifying determinates of help seeking among married women

\begin{tabular}{|c|c|}
\hline Variable label & Variable name in the dataset \\
\hline Dependent variable: Help seeking & It takes a binary form ( $1=$ Yes help seeking $)$ or failure $(0=$ No help seeking $)$ \\
\hline \multicolumn{2}{|l|}{ 1.Individual / Women characteristics } \\
\hline Maternal age & Mother's age $(1=15-24 ; 2=25-34 ; 3=35-49)$ \\
\hline Maternal religion & Maternal religion ( $1=$ Islam; $2=$ Orthodox; $3=$ catholic or others including traditional religion) \\
\hline Maternal working status & Maternal working status ( 1 = working; 2 = not working) \\
\hline Highest educational qualification & Mother education ( $1=$ Secondary and above; $2=$ Primary; $3=$ No education) \\
\hline Women's age at first marriage & Women's age at first marriage $(1=<18 ; 2=18-24 ; 3=25-34 ; 4=35-49$ years old $)$ \\
\hline Women drank alcohol & Women drinks alcohol (0 = No, 1 =Yes) \\
\hline Access to communication media & Access to at least one of communication ( $1=$ Yes; $2=\mathrm{No})$ \\
\hline Currently marital status & Currently marital status ( 1 = Currently married, 2 =Formerly married) \\
\hline Physical violence experience & Physical violence experience ( $0=\mathrm{No}, 1=\mathrm{Yes})$ \\
\hline Sexual violence experience & Sexual violence experience ( 0 =No, $1=$ Yes) \\
\hline Emotional violence experience & emotional violence experience ( $0=\mathrm{No}, 1=\mathrm{Yes})$ \\
\hline \multicolumn{2}{|l|}{ 2. Partner/Family characteristics } \\
\hline Age of husband & Husband/ Partner age ( $1=15-24 ; 2=25-35 ; 3=36-50 ; 4=>50)$ \\
\hline Duration of Marriage with current partner & Duration of Marriage (1 =0-4 Yrs; $2=5-9$ Yrs; $3=10$ Yrs and Above) \\
\hline Total number of children & Number of children $(1=0-2 ; 2=3-5 ; 3=>5)$ \\
\hline Current husband working status & Current husband working status(1=Working; 2 =not working) \\
\hline Husband education qualification & Husband education ( $1=$ Secondary and above; $2=$ Primary; $3=$ No education) \\
\hline Controlling behaviour reasoned out & Controlling behaviour mentioned ( 0 = No; $1=$ Yes) \\
\hline History of family beating & History of family beating $(0=\mathrm{No} ; 1=\mathrm{Yes})$ \\
\hline Partner drinks alcohol & Partner drinks alcohol (0 = No; 1=Yes) \\
\hline \multicolumn{2}{|l|}{ 3. Community characteristics } \\
\hline Place of residence & Place of residence (1= Urban; 2 rural) \\
\hline Geographical admin of the country & $\begin{array}{l}\text { Region (1=Tigray; } 2=\text { Afar; } 3=\text { Amhara; } 4=\text { Oromia; } 5=\text { Somali; } \\
6=\text { Benishangul-Gumuz; } 7=\text { SNNP; } 8=\text { Gambella; } 9=\text { =Harari; } 10=\text { Addis } \\
\text { Ababa; } 11=\text { Dire Dewa) }\end{array}$ \\
\hline \multicolumn{2}{|l|}{ 4. Societal characteristics } \\
\hline Wealth index & Wealth (1 = Rich; 2 = Middle; $3=$ poor $)$ \\
\hline Women decision making index & $\begin{array}{l}\text { Decision power( } 1=\text { Full power; } 2=\text { Considerable power; } 3=\text { No Power for } \\
\text { decision making) }\end{array}$ \\
\hline Attitude to wife beating & Justified for any reason ( $0=\mathrm{No} ; 1=$ Yes $)$ \\
\hline
\end{tabular}

common sources were neighbours (35.4\%), immediate family members (26.7\%) and collaborate family (12.3\%). Moreover, only a very small proportion sought assistance from a legal(police $(4.1 \%)$ or lawyer (3.6\%) source).

\section{Prevalence of help seeking by individual and partner characteristics}

The weighted prevalence of help seeking behaviour was higher among married women who had experienced physical 21.61\% [17.17, 26.83] and emotional violence

Table 2 Potential covariates used for hierarchical logistic regression model of help seeking behaviour

\begin{tabular}{llll}
\hline Model $\mathbf{1}$ & Model 2 & Model 3 & Model 4 \\
\hline Women's education, marital status, women drank & Model 1 variables, age of partner, number of & Model 2 & Model 3 variables, wealth \\
alcohol, women's access to communication & children, partner's employment status, Partner's & variables, & index, Women decision \\
media, physical violence experience emotional & education, Partner exerted controlling behaviour, & place of & making index, attitude to \\
violence & history of woman's father being physically violent & residence, & wife beating \\
& towards her mother and drank alcohol & region & \\
\hline
\end{tabular}




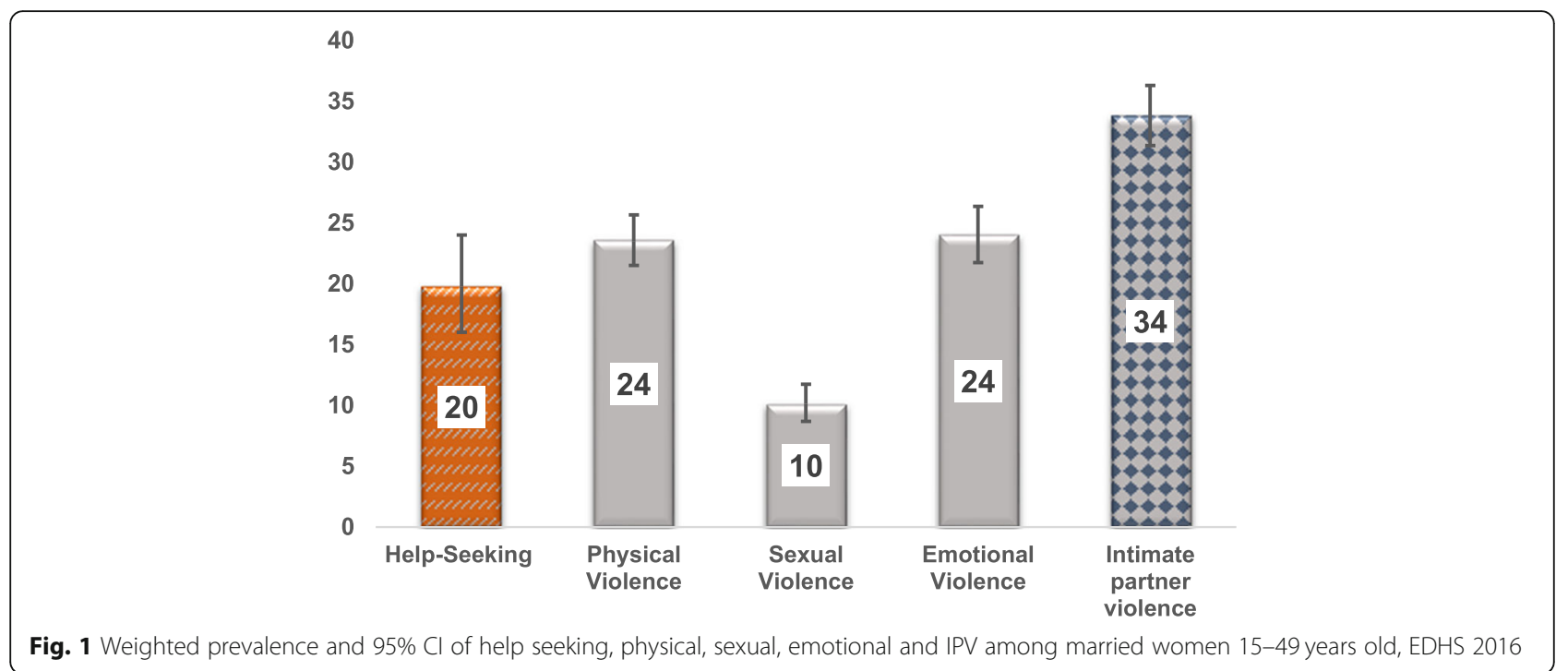

$25 \%$ [19.8, 31.49]. Additionally, women who attained at least a secondary education, were between the ages of 25-34 years old, were formerly married, and those with access to mass media (TV/radio/magazine) were more likely to engage in help seeking behaviour in Ethiopia (Table 3).

Moreover, there was a correlation between the partner characteristics of married women and help seeking behaviour. The help seeking behaviour of married women was positively linked to having an older partner, increased number of years of cohabitation, unemployment, alcohol consumption, and partners who exhibit controlling behaviour (Table 3).

\section{Prevalence of help seeking by community and social characteristics}

As presented in Table 4, the proportion of help seeking behaviour was highest amongst women residing in urban settings $36.5 \%[26.24,48.16]$, those who were from the highest wealth index $34.5 \%$ [23.61,47.39], and those women who had the skill to make decisions about their lives $25 \%$ [18.23,33.15]. In terms of regional variation, married women from Addis Ababa (42\%), Somali (27\%) and SNNP (23\%) were more likely to seek help for IPV. In contrast, married women from Benishangul-Gumuz (5.5\%), Afar (7.8\%) and Harari (10\%) were unlikely to report help seeking behaviour when they encounter violence from a partner.

\section{Multivariable analysis}

The hierarchical logistic regression model was fitted at four levels. In the final model, some of the variables that were significant in the crude association remained significant in the final model. In the final model, higher odds of help seeking behaviour was linked to physical violence, woman's educational attainment, partner's

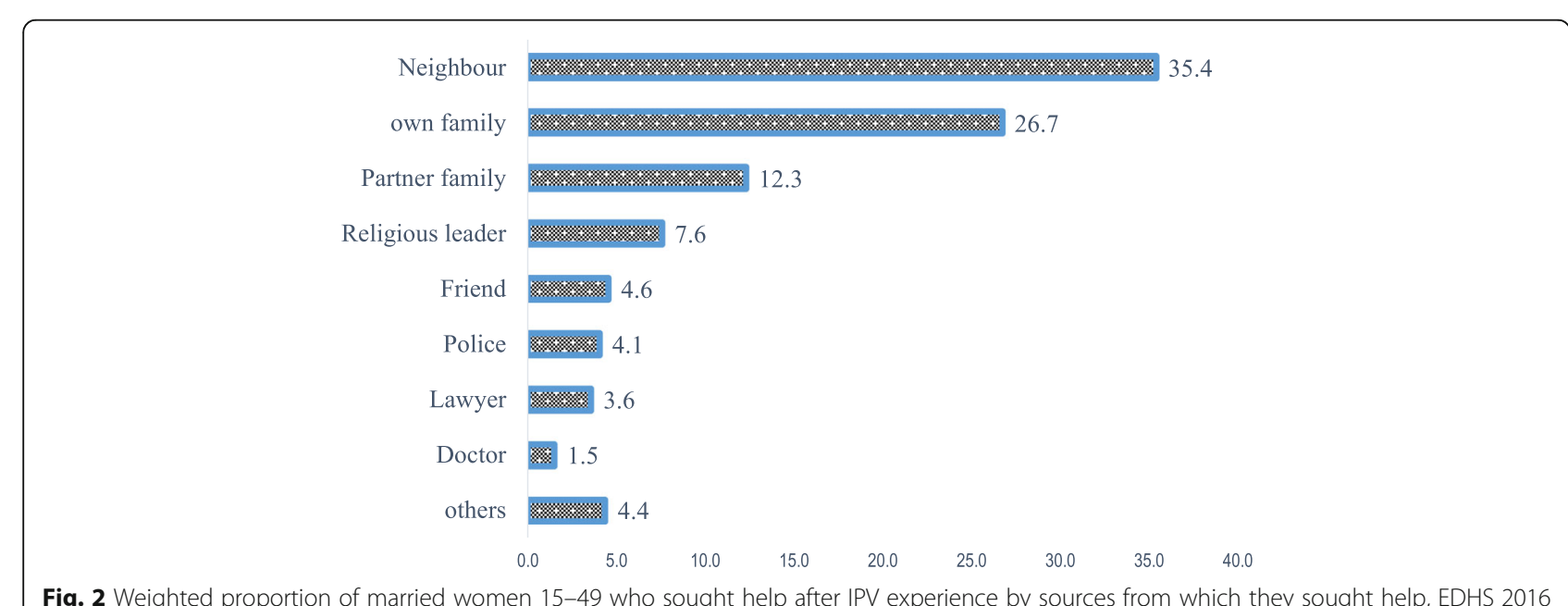

Fig. 2 Weighted proportion of married women 15-49 who sought help after IPV experience by sources from which they sought help, EDHS 2016 
Table 3 Weighted proportions ( $95 \% \mathrm{Cl}$ ) of help seeking by individual and partner level characteristics among women aged 15-49 years, EDHS 2016

\begin{tabular}{|c|c|c|c|c|}
\hline Characteristics & Options & Frequency & $\begin{array}{l}\text { Help Seeking Prevalence \% } \\
{[95 \% \mathrm{CI}]}\end{array}$ & $\begin{array}{l}P \text { - } \\
\text { Value }\end{array}$ \\
\hline \multirow[t]{2}{*}{ Physical violence } & No & 461 & $9.0[5.15,15.5]$ & 0.002 \\
\hline & Yes & 962 & $21.6[17.17,26.83]$ & \\
\hline \multirow[t]{2}{*}{ Sexual violence } & No & 1065 & $19.1[14.46,24.86]$ & 0.681 \\
\hline & Yes & 358 & $20.8[14.8,28.49]$ & \\
\hline \multirow[t]{2}{*}{ Emotional violence } & No & 370 & $9.8[6.47,14.76]$ & $<0.001$ \\
\hline & Yes & 1053 & $25.2[19.8,31.49]$ & \\
\hline \multicolumn{5}{|l|}{ Women factors } \\
\hline \multirow[t]{3}{*}{ Age (Years) } & $15-24$ & 295 & $10.0[6.156,15.70]$ & 0.003 \\
\hline & $25-34$ & 610 & $24.3[18.4,31.25]$ & \\
\hline & $35-49$ & 518 & $20.5[14.97,27.41]$ & \\
\hline \multirow[t]{3}{*}{ Women education } & Secondary and above & 839 & $34.4[21.08,50.72]$ & 0.030 \\
\hline & Primary & 413 & $15.1[10.75,20.81]$ & \\
\hline & No education & 171 & $20.0[14.88,26.27]$ & \\
\hline \multirow[t]{3}{*}{ Women religion } & Muslim & 510 & $18.2[11.73,27.16]$ & 0.510 \\
\hline & Orthodox & 589 & $22.2[16.38,29.34]$ & \\
\hline & $\begin{array}{l}\text { Other (Catholic/ } \\
\text { traditional) }\end{array}$ & 324 & $17.8[12.02,24.2]$ & \\
\hline \multirow[t]{2}{*}{ Women working status } & working & 567 & $21.6[16.7,27.53]$ & 0.460 \\
\hline & Not working & 856 & $18.7[13.72,25.12]$ & \\
\hline \multirow[t]{2}{*}{ Women's age at first marriage (years) } & 18 and above & 541 & $17.2[12.63,22.93]$ & 0.190 \\
\hline & $<18$ & 882 & $21.2[16.51,26.59]$ & \\
\hline \multirow[t]{2}{*}{ Number of union/partner women have } & One & 1145 & $20.12[15.56,25.62]$ & 0.710 \\
\hline & More than One & 278 & $18.5[12.3,26.81]$ & \\
\hline \multirow[t]{2}{*}{ Current marital status } & Currently married & 1190 & $18.2[14.19,22.94]$ & 0.041 \\
\hline & Formerly married & 233 & $27.4[19.05,37.62]$ & \\
\hline \multirow[t]{2}{*}{ Woman ever drank alcohol } & No & 886 & $17.6[13.15,23.08]$ & 0.180 \\
\hline & Yes & 537 & $23.3[16.81,30.93]$ & \\
\hline \multirow[t]{2}{*}{ Access to communication media } & Yes & 395 & $23.5[15.91,33.22]$ & 0.020 \\
\hline & No & 1028 & $18.7[14.11,24.27]$ & \\
\hline \multicolumn{5}{|l|}{ Partners variables } \\
\hline \multirow[t]{4}{*}{ Age of partner (years) } & $15-24$ & 36 & $10[2.845,28.72]$ & 0.003 \\
\hline & $25-35$ & 313 & $11.2[7.336,16.8]$ & \\
\hline & $36-50$ & 441 & $22.9[16.52,30.95]$ & \\
\hline & $50+$ & 333 & $25.4[19.12,32.8]$ & \\
\hline \multirow[t]{3}{*}{ Duration of marriage (years) } & $0-4$ & 221 & $10.4[5.827,17.99]$ & 0.008 \\
\hline & $5-9$ & 277 & $15.3[9.852,23.1]$ & \\
\hline & 10 and above & 925 & $23.5[18.21,29.79]$ & \\
\hline \multirow[t]{3}{*}{ Partner education } & Secondary and above & 541 & $13.2[6.78,24.04]$ & 0.230 \\
\hline & Primary & 506 & $15.7[11.49,20.98]$ & \\
\hline & No Education & 376 & $20.9[14.66,28.89]$ & \\
\hline \multirow[t]{2}{*}{ Husband working status } & employed & 1084 & $17.1[13.23,21.85]$ & 0.015 \\
\hline & unemployed & 339 & $27.8[19.71,37.74]$ & \\
\hline Number of children & $0-2$ & 198 & $15.5[11.03,21.34]$ & 0.263 \\
\hline
\end{tabular}


Table 3 Weighted proportions (95\% Cl) of help seeking by individual and partner level characteristics among women aged 15-49 years, EDHS 2016 (Continued)

\begin{tabular}{|c|c|c|c|c|}
\hline Characteristics & Options & Frequency & $\begin{array}{l}\text { Help Seeking Prevalence \% } \\
{[95 \% \mathrm{Cl}]}\end{array}$ & $\begin{array}{l}P \text { - } \\
\text { Value }\end{array}$ \\
\hline & 3 to 5 & 427 & $21.2[15.84,27.88]$ & \\
\hline & $5+$ & 553 & $23.3[14.8,34.65]$ & \\
\hline \multirow[t]{2}{*}{ Husband has controlling behaviour } & No & 334 & $10.5[6.274,17.13]$ & 0.005 \\
\hline & Yes & 1089 & $22.1[17.49,27.47]$ & \\
\hline \multirow{2}{*}{$\begin{array}{l}\text { History of woman's father being physically violent towards her } \\
\text { mother }\end{array}$} & No & 803 & $18[14.05,22.82]$ & 0.301 \\
\hline & Yes & 620 & $21.7[15.8,28.94]$ & \\
\hline \multirow[t]{2}{*}{ Partner drank alcohol } & No & 856 & $15.4[10.33,22.39]$ & 0.017 \\
\hline & Yes & 567 & $25.2[20.33,30.88]$ & \\
\hline
\end{tabular}

unemployment, controlling behaviour, partner's alcohol consumption, regional variability, and women of high wealth index (Table 5).

For instance, women who experienced physical violence were three times more likely to seek help as compared to those who did not experience physical violence. Married women who attained a primary education had
$29 \%$ higher odds of help seeking behaviour as compared to women with no education. Additionally, women with partners who exhibit controlling behaviour and consume alcohol had higher odds of reporting help seeking behaviour. Women from urban and higher economic wellbeing settings, such as Addis Ababa, had higher odds of seeking help.

Table 4 Weighted Prevalence of help seeking by community and societal level among married women aged 15-49 years, EDHS 2016

\begin{tabular}{|c|c|c|c|c|}
\hline Characteristics & Options & Frequency & Help-Seeking Prevalence $\%[95 \% \mathrm{Cl}]$ & $P$-value \\
\hline \multicolumn{5}{|l|}{ Community Factors } \\
\hline \multirow[t]{2}{*}{ Place of residence } & Urban & 335 & $36.5[26.24,48.16]$ & $<0.001$ \\
\hline & Rural & 1088 & $17.0[12.95,22.07]$ & \\
\hline \multirow[t]{11}{*}{ Geography (region) } & Tigray & 162 & $20.3[12.56,31.1]$ & 0.128 \\
\hline & Afar & 81 & $7.8[2.43,22.21]$ & \\
\hline & Amhara & 186 & $19.2[13.54,26.58]$ & \\
\hline & Oromia & 253 & $17.6[11.14,26.7]$ & \\
\hline & Somali & 47 & $28.6[15.1,47.31]$ & \\
\hline & Benishangul-Gumuz & 134 & $5.5[1.93,14.86]$ & \\
\hline & SNNP & 166 & $23.8[16.78,32.68]$ & \\
\hline & Gambella & 120 & $13.3[7.90,21.65]$ & \\
\hline & Harrari & 109 & $10[4.56,20.93]$ & \\
\hline & Addis Ababa & 78 & $42.2[28.76,56.94]$ & \\
\hline & Dire Dawa & 87 & $18.6[10.3,31.29]$ & \\
\hline \multicolumn{5}{|l|}{ Societal factors } \\
\hline \multirow[t]{3}{*}{ Wealth index } & rich & 634 & $34.5[23.61,47.39]$ & 0.003 \\
\hline & middle & 229 & $16.3[10.65,24.06]$ & \\
\hline & Poor & 352 & $15.7[10.72,22.5]$ & \\
\hline \multirow[t]{3}{*}{ Decision making Power } & Full power & 364 & $25[18.23,33.15]$ & 0.204 \\
\hline & Considerable power & 253 & $21[12.48,33.17]$ & \\
\hline & No power & 806 & $17.0[12.71,22.45]$ & \\
\hline \multirow[t]{2}{*}{ Attitudes of wife beating } & not justified & 492 & $24.8[16.89,34.98]$ & 0.110 \\
\hline & justified & 931 & $17.6[13.74,22.27]$ & \\
\hline Overall & & 1423 & $19.75[15.9 ; 24.3]$ & \\
\hline
\end{tabular}


Table 5 Multivariable analysis of help seeking fitted by four level covariates among married women aged 15-49 years after experienced IPV, EDHS 2016

\begin{tabular}{|c|c|c|c|c|c|}
\hline Help Seeking factors & Option & COR & AOR & {$[95 \% \mathrm{Cl}]$} & $P$-value \\
\hline \multirow[t]{2}{*}{ Physical violence } & No & 1.00 & 1.00 & & \\
\hline & Yes & $2.76[1.42 ; 5.41]$ & 3.22 & {$[1.12,9.20]$} & $0.029^{*}$ \\
\hline \multirow[t]{2}{*}{ Sexual Violence } & No & 1.00 & 1.00 & & \\
\hline & Yes & $1.12[0.66 ; 1.87]$ & 1.36 & {$[0.75,2.44$} & 0.307 \\
\hline \multirow[t]{2}{*}{ Emotional violence } & No & 1.00 & 1.00 & & \\
\hline & Yes & $3.11[1.76 ; 5.36]$ & 1.96 & {$[0.92,4.20]$} & 0.082 \\
\hline \multirow[t]{3}{*}{ Maternal education } & No education & 1.00 & 1.00 & & \\
\hline & Primary & $0.71[0.18 ; 0.43]$ & 0.16 & {$[0.03,0.82]$} & $0.026^{*}$ \\
\hline & Secondary and above & $2.10[0.06 ; 0.98]$ & 0.21 & {$[0.04,1.21]$} & 0.080 \\
\hline \multirow[t]{2}{*}{ Women drink alcohol } & No & 1.00 & 1.00 & & \\
\hline & Yes & $1.42[0.83 ; 2.34]$ & 0.98 & {$[0.43,2.21]$} & 0.952 \\
\hline \multirow[t]{2}{*}{ Having access any means of communication } & Yes & 1.00 & 1.00 & & \\
\hline & No & $0.75[0.41 ; 1.37]$ & 1.62 & {$[0.61,4.26]$} & 0.329 \\
\hline \multirow[t]{4}{*}{ Age of partner } & $15-24$ & 1.00 & 1.00 & & \\
\hline & $25-35$ & $0.1 .2[0.29 ; 4.67]$ & 0.51 & {$[0.12,2.20]$} & 0.367 \\
\hline & $36-50$ & $2.7[0.69 ; 10.8]$ & 0.91 & {$[0.21,3.84]$} & 0.894 \\
\hline & $50+$ & $3.12[0.80 ; 12.13]$ & 0.75 & {$[0.15,3.89]$} & 0.736 \\
\hline \multirow[t]{3}{*}{ Number of children } & $0-2$ & 1.00 & 1.00 & & \\
\hline & 3 to 5 & $1.5[0.84 ; 2.5]$ & 2.07 & {$[0.94,4.52]$} & 0.069 \\
\hline & $5+$ & $1.7[0.84 ; 3.30]$ & 2.21 & {$[0.85,5.70]$} & 0.103 \\
\hline \multirow[t]{2}{*}{ Husband working status } & Employed & 1.00 & 1.00 & & \\
\hline & unemployed & $1.9[1.12 ; 3.11]$ & 3.71 & {$[1.29,10.65]$} & $0.015^{*}$ \\
\hline \multirow[t]{3}{*}{ Partner education } & Secondary and above & 1.00 & & & \\
\hline & Primary & $1.22[0.54 ; 2.76]$ & 1.96 & {$[0.50,7.63]$} & 0.331 \\
\hline & No Education & $1.74[0.75 ; 4.03]$ & 4.09 & {$[0.96,17.37]$} & 0.056 \\
\hline \multirow[t]{2}{*}{ Partner's controlling behaviour } & No & 1.00 & & & \\
\hline & Yes & $2.4[1.30 ; 4.51]$ & 3.24 & {$[1.45,7.22]$} & $0.004^{*}$ \\
\hline \multirow{2}{*}{$\begin{array}{l}\text { History of woman's father being physically } \\
\text { violent towards her mother }\end{array}$} & No & 1.00 & & & \\
\hline & Yes & $1.3[0.81 ; 1.95]$ & 1.15 & {$[0.65,2.05]$} & 0.626 \\
\hline \multirow[t]{2}{*}{ Partner ever drinks alcohol } & No & 1.00 & & & \\
\hline & Yes & $1.92[1.12 ; 3.08]$ & 2.45 & {$[1.23,4.86]$} & $0.011^{*}$ \\
\hline \multirow[t]{2}{*}{ Place of residence } & Urban & 1.00 & & & \\
\hline & Rural & $0.34[0.20 ; 0.63]$ & 1.67 & {$[0.43,6.49]$} & 0.456 \\
\hline \multirow[t]{10}{*}{ Region/Geography } & Tigray & 1.00 & & & \\
\hline & Afar & $0.33[0.08 ; 1.2]$ & 0.54 & {$[0.06,4.65]$} & 0.576 \\
\hline & Amhara & $0.93[0.46 ; 1.90]$ & 2.68 & {$[0.82,8.74]$} & 0.101 \\
\hline & Oromia & $0.840[.38 ; 1.83]$ & 1.76 & {$[0.53,5.87]$} & 0.354 \\
\hline & Somali & $1.56[0.58 ; 4.23]$ & 4.70 & {$[0.98,22.57]$} & 0.054 \\
\hline & Benishangul-Gumuz & $0.23[0.07 ; 0.79]$ & 0.23 & {$[0.02,2.61]$} & 0.233 \\
\hline & SNNP & $1.22[0.59 ; 2.52]$ & 4.16 & {$[1.20,14.48]$} & $0.025^{*}$ \\
\hline & Gambella & $0.6[0.26 ; 1.37]$ & 1.71 & {$[0.43,6.84]$} & 0.444 \\
\hline & Harrari & $0.44[0.15 ; 1.23]$ & 1.97 & {$[0.36,10.88]$} & 0.436 \\
\hline & Addis Ababa & $2.86[1.25 ; 6.54]$ & 4.76 & {$[0.84,26.99]$} & 0.078 \\
\hline
\end{tabular}


Table 5 Multivariable analysis of help seeking fitted by four level covariates among married women aged 15-49 years after experienced IPV, EDHS 2016 (Continued)

\begin{tabular}{|c|c|c|c|c|c|}
\hline Help Seeking factors & Option & COR & AOR & {$[95 \% \mathrm{Cl}]$} & $P$-value \\
\hline & Dire Dawa & $0.89[0.36 ; 2.19]$ & 3.02 & {$[0.62,14.76]$} & 0.172 \\
\hline \multirow[t]{3}{*}{ Wealth index } & poor & 1.00 & & & \\
\hline & middle & $1.04[0.53 ; 2.0]$ & 0.75 & {$[0.37,1.53]$} & 0.430 \\
\hline & Rich & $2.8[1.41 ; 5.64]$ & 4.13 & {$[1.29,13.20]$} & $0.017^{*}$ \\
\hline \multirow[t]{3}{*}{ Decision making } & Full power & 1.00 & & & \\
\hline & Considerable power & $0.83[0.42 ; 1.54]$ & 1.45 & {$[0.51,4.17]$} & 0.488 \\
\hline & No power & $0.62[0.36 ; 1.04]$ & 0.85 & {$[0.34,2.15]$} & 0.734 \\
\hline \multirow[t]{2}{*}{ Wife beating } & not justified & 1.00 & & & \\
\hline & justified & $0.65[0.37 ; 1.12]$ & 1.07 & {$[0.56,2.07]$} & 0.836 \\
\hline
\end{tabular}

* Significant covariates at $P<0.05$

Geographic variation and spatial clustering of help seeking behaviour among married women who experienced IPV In this study, low prevalence or cold spot areas of IPV were reported in Somalia, Afar, and parts of the Eastern Amhara region. High IPV spots were observed in Western and Central Oromia, Western Amhara, Central Tigray, Eastern Benishangul-Gumuz, Gambella, Dire-Dawa and Harari (Fig. 3). The spatial distribution of IPV showed significant geographic variation among regions in Ethiopia (Moran's I index $=3.15 ; \mathrm{Z}$ score $=47.3 ; \mathrm{P}<$ 0.001).
The prevalence of reported help seeking behaviour was high (42\%) in Addis Ababa while the lowest was in the developing regions of Ethiopia, especially in Afar, Gambella and Benishangul Gumuz regions (Fig. 4).

The Getis-Ord $\mathrm{Gi}^{*}$ statistics showed that the prevalence of reported help seeking behaviour among women had statistically significant variation among regions in Ethiopia. Hot spots of help seeking behaviour among married women who experienced IPV were clustered predominantly around Addis Ababa, Northeastern SNNPR, Central Oromia and Somali. In contrast,

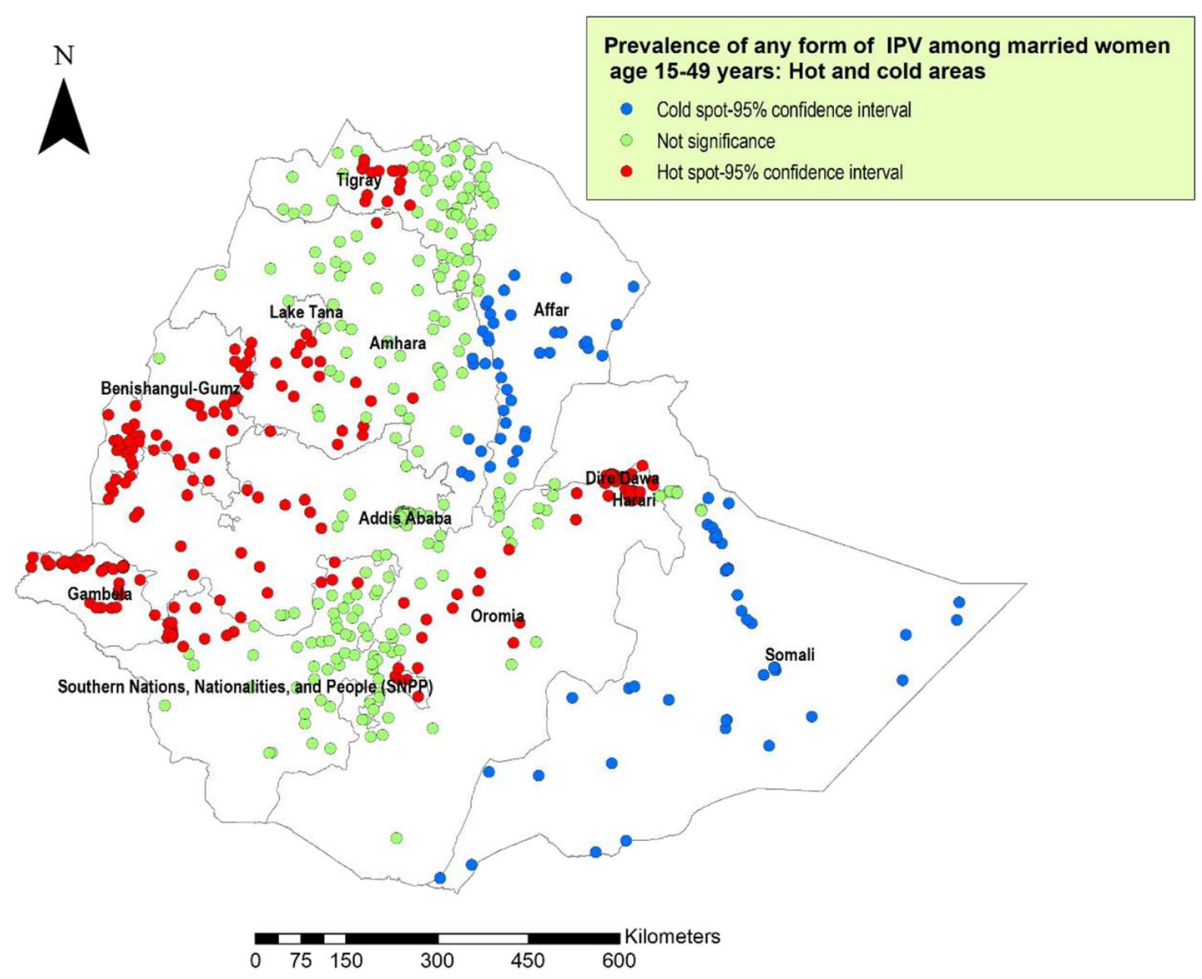

Fig. 3 Map of prevalence of IPV among married women age 15-49 years in Ethiopia; EDHS 2016 (additional Fig. 3) 


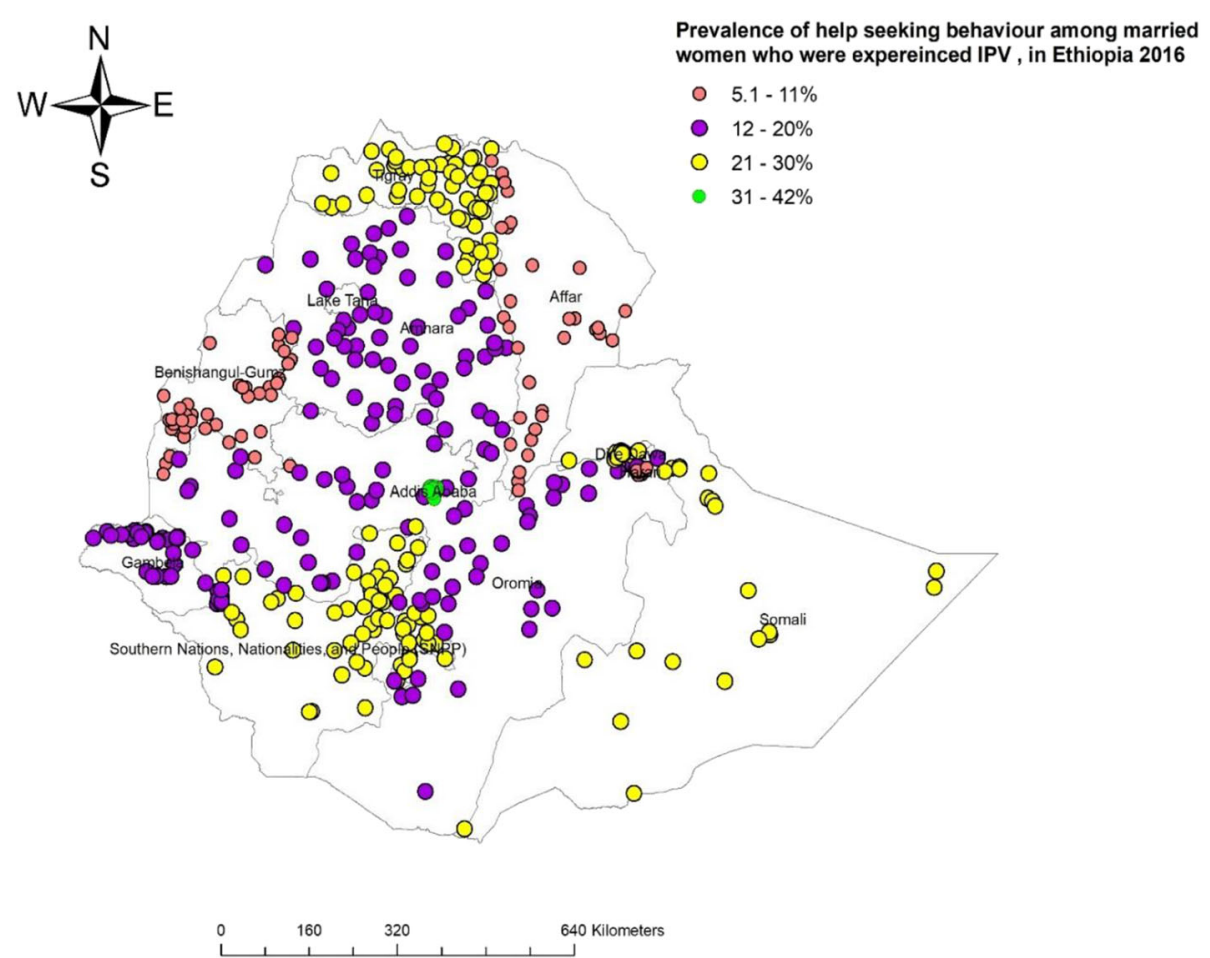

Fig. 4 Map of prevalence help seeking behaviour among married women age 15-49years, EDHS 2016 (additional Fig. 4)

Benishangul-Gumz, Eastern and Western Amhara, Harari, and Afar were cold spots, despite high prevalence of IPV in these regions (Fig. 5).

\section{Discussion}

The aim of this study was to identify any geographical variation in help seeking behaviour among married women ages $15-49$ years old in Ethiopia and to investigate its associated factors using 2016 EDHS survey data.

Overall, a low prevalence of reported help seeking behaviour in Ethiopia was identified among married women who experienced IPV. This was consistent with other studies conducted in similar settings including in Ethiopia and other SSA countries [2, 33, 34]. It is important to note that the majority of the women who reported help seeking behaviour were seeking support from informal sources such as neighbours, families, or friends. This indicates that IPV in Ethiopia is commonly managed informally at the interpersonal and community levels. Due to the limited training and capacity of informal sources, IPV remains high and in need of intervention. The low prevalence of help seeking behaviour might be related to the socially accepted dominance of husbands over their households, including their spouses. This unequal distribution of power and status in domestic relationships is an important indicator of the dominant cultural and social norms of Ethiopian society [2, 35-39]. Moreover, low prevalence may be influenced by the disparate socio-demographic characteristics of women in Ethiopia, such as low educational attainment. Other factors associated include the type of violence experienced and partner related characteristics, such as controlling behaviour, alcohol consumption, employment status, and wealth of the family. This is consistent with studies conducted in various areas [40-42].

Generally, the prevalence of help seeking behaviour in Ethiopia was very low at $19.75 \%$. The use of spatial analysis showed that the phenomenon was heterogeneous in the various regions of Ethiopia. It also identified clusters of reported help seeking behaviour among women who experienced IPV, mainly in Addis Ababa, North Eastern SNNPR, Central Oromia, and the Somali regions. In contrast, married women in Benishangul-Gumuz, Gambella, East and Western Amhara and Afar regions had the lowest odds of seeking help when they experienced IPV. The geographical variation of IPV in the regions of Ethiopia might be related with the patriarchal nature of community that promotes male dominance and the deep rooted acceptance of violence among women $[2,18]$. Moreover, in some of the regions such as Gambella, Afar and other Western Oromia, there are large number of refuges and internally displaced peoples or conflict and displacement disrupt all aspects of life. In addition, there are many peoples who are travelled to Oromia and Benshangule region and often separated from their family and loved ones. This refugees may experience significant 


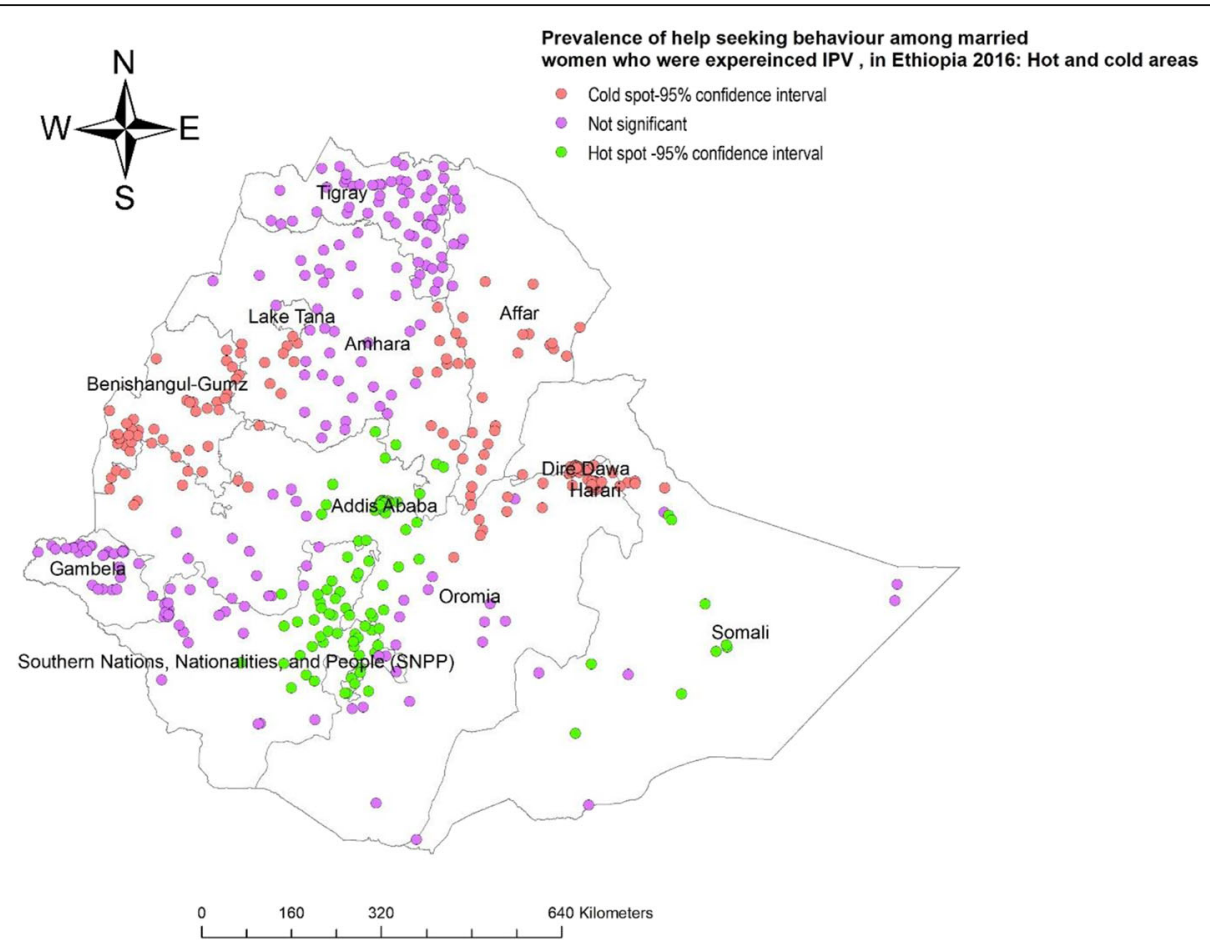

Fig. 5 Map of hot and cold areas of help seeking behaviour among married women age 15-49 years old, EDHS 2016 (additional Fig. 5)

distrust and discrimination in the host community of the refuges and migrants confidence to have help seeking behaviour due to fear to new environment and lack of the confidence the access the services $[8,18,23]$.

Moreover, the decision to seek help, from either a formal or informal source of support, depends how victims problematize their abusive situation. The findings of this study indicate that women experiencing physical violence is the most significant reason to seek help. This might be attributed to the severity of the injury and pain which may require immediate need of services or support. Moreover, culturally, it is considered less shameful to report IPV to family rather than a formal source. This may be why it was more common for women to report seeking help from informal sources [40, 43].

Attaining a secondary or above education was found to be the most important factor to enhancing help seeking behaviours of women and reducing the occurrence of IPV. In this study, the odds of reporting help seeking behaviour was higher amongst women who have a secondary or above education compared to women who have no education. Studies indicate that education informs women to better be able to identify and mitigate IPV. It also increases the help seeking behaviour of women [37, 44-47]. Lower levels of education attainment might be related to a lack of understanding around the mitigation measures, limited exposure to legal support, and increased acceptance of traditional beliefs which are harmful to women. Moreover, it might also be correlated to other factors such as resources and decision-making power [48]. Health behaviour theory demonstrates that education (increased knowledge and awareness) is a strong means for improved behaviour $[37,49,50]$. Education is a way of informing people of the normative underpinnings of a society and can expose them to global discourses rejecting IPV and encouraging help seeking behaviour $[3,51]$.

This study shows that partner characteristics are important predictors for women seeking help in response to IPV. More specifically, a partners' employment status, controlling behaviour, and alcohol consumption were strongly associated with reported help seeking behaviour among married women who experienced IPV in Ethiopia [52].

The interdependence between economic dependence of women and low help seeking behaviour is well documented in various studies $[36,53,54]$. When the partners of women experiencing IPV were unemployed, the likelihood of women reporting the violence was doubled. This suggests women experiencing IPV may leave a dangerous relationship when there is less economic dependence on their partners [55-57].

According to feminist theory, controlling behaviour within relationships is related to unequal social distribution of power, which is rampant in patriarchal societies. This revealed the societal-level power differences within societies that work directly or/and indirectly to endorse a male-dominated social order and family structure. This often result in men exercising power and control over 
women in several ways, one of which is IPV and the likelihood of help seeking controlled [58, 59]. In contrast, this study found that women with partners exhibiting controlling behaviour were more likely to look for help from formal or informal sources. Although additional research is needed, these findings may indicate that the controlling behaviour of an intimate partner and the use of physical and emotional threats can result in such adverse effects that increase the help seeking behaviour of women $[58,60]$. As a result, focusing more attention on understanding the associated factors of controlling behaviour of partners may help to design targeted interventions increase the help seeking behaviour of women.

The strong link found between partner alcohol consumption and increased help seeking behaviour may be attributed to the potential for alcohol to affect the thinking, perceptions and risk-taking of male partners; this may create a fear or increased violence in women which leads to reporting the violence to someone who can provide support $[61,62]$.

Additionally, this study revealed wealth increases the odds of help-seeking behaviour among women in Ethiopia. This is consistent with previous research which suggests that women who earn higher incomes or who are financially independent are more likely to seek help [63]. Women who live in poverty or low income families are less likely to seek help [64, 65]. Overall, this influence of wealth on help seeking behaviour may be because access to financial resources widens the range of support and services available.

\section{Implication for programming and policy}

Findings reported in this study provide vital evidence to inform programs and policy, and to guide investment in woman's health to prevent and reduce the consequences of IPV in Ethiopia, in alignment with the 2030 SDG five target that includes target to eliminate all forms of violence against all women and girls including trafficking, sexual and other types of exploitation and SDG 16 target related to significantly reduce all forms of violence and related death rates everywhere and to end abuse, exploitation, trafficking and all forms of violence against children. This study provides insight to the required response to women experiencing IPV by identifying the geographical hot spots in Ethiopia and associated factors with help seeking behaviour. Governmental policy should prioritize the prevention of IPV and increase the demand for formal services by women, especially given the high prevalence of IPV and low help seeking behaviours in all regions of Ethiopia. This strategy needs to be supported by a legal framework to accommodate social support that includes power equality, women's economic empowerment, and provision of health information and services.

\section{Strength and limitation of the study}

This study used the golden standard measurement for most developing countries (DHS measure), EDHS 2016, a representative survey which is the largest dataset in Ethiopia. The study team used multistage cluster sampling and analysis, and geospatial analysis identify hotspot areas across the country. A limitation of this study is the cross-sectional nature of the study design which may affect causality. Moreover, since the study is selfreported, recall bias may be present and underreported due to fear of stigma and discrimination and cannot be validated by formal and informal sources. Additionally, this study only limited to EDHS questionaries and lacks some variables related to refugees, internally displaced people and many other priorities.

\section{Conclusions and recommendations}

In summary, the findings show that the prevalence of help seeking behaviour among married women who experienced IPV in Ethiopia was very low, although IPV is highly prevalent. The lowest help seeking behaviour prevalence was reported in Benishangul-Gumuz, Western and Eastern Amhara, Gambella and Afar regions. Formal help seeking behaviour in Ethiopia is limited, which poses a challenge to designing effective public health interventions to address IPV. IPV is underreported to formal sources such as police, courts, and other social service institutions. Factors associated with poor help seeking behaviour among married women include low educational attainment, the type of violence experienced, husband controlling behaviour, higher alcohol consumption, partner's unemployment status, geographical location, and poor wealth level of the household and surrounding community. Moreover, the findings clearly show that intervention requires a multisectoral response including education for capacity building and to create awareness of the women and communities, economic sector for creating business opportunities, health sector to avail services and counsel clients, and legal sector for law enforcement are some of the sector offices that needs to be involved in response to IPV and help seeking. Building the capacity of health care providers in response to psychosocial and gender based violence support. Governmental policies should focus on the prevention of IPV through various means such as increasing demand for women to access formal services (health, legal and social services), if they experienced IPV. Additionally, the help seeking and IPV prevention strategy needs to be supported by a legal framework and law enforcement to accommodate the social support offered in a given community. Future studies should focus on qualitative research that centres an in-depth understanding of gender inequity due to male dominance. Additionally, more research that focuses on interventions to improve women's reporting of IPV is needed. Moreover, further 
study that focus on help-seeking behaviours difference in sub-groups such as refugees, ethnic and religious minorities are some research areas.

\section{Authors' contributions}

MDM was involved in the conceptualization, analysed and write the report of this study. YWA and MWM authors reviwed and contributed in the analysis and write up the manuscript. All the were involved in the revision and editing of the manuscript. The author(s) read and approved the final manuscript.

\section{Funding}

This study has not secured any financial support.

\section{Ethics approval and consent to participate}

This study used secondary data that are available in the public domain from EDHS. The institutions that commissioned, funded, or managed the surveys addressed ethical procedures. All DHS surveys are approved by the Inner City Fund (ICF) international and an institutional review board in specific countries to ensure that they comply with the United States Department of Health and Human Services' regulations for the protection of human subjects.

\section{Consent for publication}

Not applicable.

\section{Competing interests}

The authors declare that they have no competing interests.

\section{Author details}

${ }^{1}$ Monitoring Evaluation and Research Department, Amref Health Africa in Ethiopia, Addis Ababa, Ethiopia. ${ }^{2}$ School of Nursing and Midwifery, Western Sydney University, Sydney, Australia. ${ }^{3}$ Faculty of Public Health, GAMBY College of Medical Science, Addis Ababa, Ethiopia. ${ }^{4}$ School of Medicine and Public Health, Faculty of Health and Medicine, University of Newcastle, Newcastle, Australia.

Received: 3 August 2020 Accepted: 10 December 2020

Published online: 06 January 2021

\section{References}

1. Krug EG, et al. World report on violence and health. Geneva: World Health Organization; 2002.

2. WHO. Global and regional estimates of violence against women. Prevalence and health effects of intimate partner violence and non-partner sexual violence. WHO; 2013. https:/www.who.int/publications/i/item/ 9789241564625.

3. Muluneh MD, Stulz V, Francis L, Agho K. Gender Based Violence against Women in Sub-Saharan Africa: A Systematic Review and Meta-Analysis of Cross-Sectional Studies. Int J Environ Res Public Health. 2020;17(3):903 2020.

4. World Health Organization (WHO). Understanding and addressing violence against women. Geneva: 2012. https://apps.who.int/iris/bitstream/handle/1 0665/77433/WHO_RHR_12.35_eng.pdf;jsessionid=89E373A47A16FE51E77C5 B9A817E4438? sequence $=1$.

5. Garcia-Moreno C, et al. Prevalence of intimate partner violence: findings from the WHO multi-country study on women's health and domestic violence. Lancet. 2006;368(9543):1260-9.

6. Ziaei S, Naved RT, Ekstrom EC. Women's exposure to intimate partner violence and child malnutrition: findings from demographic and health surveys in Bangladesh. Matern Child Nutr. 2014;10(3):347-59.

7. Sarkar NN. The impact of intimate partner violence on women's reproductive health and pregnancy outcome. J Obstet Gynaecol. 2008;28(3) 266-71

8. Central Statistical Agenecy (CSA). Ethiopia Demographic and Health Survey 2011 report. Addis Ababa, Ethiopia and Calverton, Maryland, USA: 2011. https://dhsprogram.com/pubs/pdf/PR10/PR10.pdf.

9. WHO. Global and Regional Estimates of Violence against Women: Prevalence and Health Effects of Intimate Partner Violence and Nonpartner Sexual Violence. Geneva: 2013. https://apps.who.int/iris/bitstream/handle/1 0665/85239/9789241564625_eng.pdf?sequence=1.
10. Ansara DL, Hindin MJ. Formal and informal help-seeking associated with women's and men's experiences of intimate partner violence in Canada. Soc Sci Med. 2010;70:1011-8. https://doi.org/10.1016/j.socscimed.2009.12.009.

11. Rowan $\mathrm{K}$, Mumford $\mathrm{E}$, Clark CJ. Women's empowerment associated with help-seeking for spousal violence in India? I Interpers Violence. 2015: 886260515618945

12. Morrison KE, Luchok KJ, Richter DL, Parra-Medina D. Factors influencing help-seeking from informal networks among African American victims of intimate partner violence. J Interpers Violence. 2006;21(11):1493-511. https:// doi.org/10.1177/0886260506293484.

13. Paul S. Intimate Partner Violence and Women's Help-seeking Behaviour: Evidence from India. Journal of Interdisciplinary Economics. 2016;28(1):53-82. https://doi.org/10.1177/0260107915609818.

14. Kavitha VRS. Spousal domestic violence of married women in India. J Sociol Soc Anthropol. 2012;3(1):7-13.

15. Coker AL, et al. Social support protects against the negative effects of partner violence on mental health. J Womens Health Gend Based Med. 2002;11(5):465-76

16. Dessalegn $\mathrm{M}$, et al. Gender inequality and the sexual and reproductive health status of young and older women in the Afar region of Ethiopia. Int J Environ Res Public Health. 2020:17(12):4592.

17. Kimuna SR, Djamba YK, Ciciurkaite G, Cherukuri S. Domestic violence in India: insights from the 2005-2006 national family health survey. J Interpers Violence. 2013;28(4):773-807. https://doi.org/10.1177/ 0886260512455867.

18. Jewkes R, Levin J, Penn-kekana L. Risk factors for domestic violence: findings from a south African cross-sectional study. Soc Sci Med. 2002;55:1603-17.

19. Berhanie $\mathrm{E}$, et al. Intimate partner violence during pregnancy and adverse birth outcomes: a case-control study. Reprod Health. 2019;16:9.

20. Federal Democratic Republic of Ethiopia, The Revised Family Code, in Federal Negarit Gazetta Extra Ordinary Issue No. 1/2000 The Revised Family Code Proclamation No. 213/2000. The Revised Family Code.

21. Federal Democratic Republic of Ethiopian, The Criminal Code of the Federal Democratic Republic of Ethiopian, in Proclamation No.414/2004. 2004.

22. Ricardo Hausmann, Laura D. Tyson, and Saadia Zahidi. The global gender gap report 2010 2010; Available from: https://www.weforum.org/reports/ global-gender-gap-report-2010.

23. CSA. Ethiopia Demographic and Health Survey. Calverton, Maryland, USA: Addis Ababa, Ethiopia; 2016. https://dhsprogram.com/methodology/survey/ survey-display-478.cfm

24. Misganaw A, Haregu TN, Deribe K, et al. National mortality burden due to communicable, non-communicable, and other diseases in Ethiopia, 19902015: findings from the Global Burden of Disease Study 2015. Popul Health Metrics. 2017;15:29. https://doi.org/10.1186/s12963-017-0145-1.

25. WHO, et al., Maternal mortality in 1990-2015. 2015.

26. FMOH. Health Sector Transfromation Plan (HTSP) 2015/2016-2019/2020. Addis Ababa: FMOH; 2015.

27. WHO, Mental ealth: New understanding, New ope. 2001.

28. Measure DHS. Available from: https://www.dhsprogram.com/data/availabledatasets.cfm.

29. Hosmer DW, Lemeshow S. Applied logistic regression. New York: Wiley; 2000

30. Esri. How Spatial Autocorrelation (Global Moran's I) works. [cited 2020 July 7]; Available from: https://pro.arcgis.com/en/pro-app/tool-reference\%20/ spatial-statistics/h-how-spatial-autocorrelation-moran-s-i-spatial-st.htm\#: : text=The\%20Spatial\%20Autocorrelation\%20(Global\%20Moran's,clustered\%2 C\%20dispersed\%2C\%20or\%20random.

31. Esri. How High/Low Clustering (Getis-Ord General G) works; Available from: https:/desktop.arcgis.com/en/arcmap/10.3/tools/spatial-statistics-toolbox/hhow-high-low-clustering-getis-ord-general-g-spat.htm\#: :text=The\%20High\%2 FLow\%20Clustering\%20(Getis,spatial\%20clustering\%20of\%20feature\%20values.

32. Ord JK, Getis A. Local spatial autocorrelation statistics: distributional issues and an application. Geogr Anal. 1995;27:286-306.

33. Yenealem DG, et al. Violence at work: determinants \& prevalence among health care workers, northwest Ethiopia: an institutional based cross sectional study. Ann Occup Environ Med. 2019;31:8.

34. Gebrezgi $\mathrm{BH}$, et al. Factors associated with intimate partner physical violence among women attending antenatal care in Shire Endaselassie town, Tigray, northern Ethiopia: a cross-sectional study, July 2015. Reprod Health. 2017;14:10.

35. Decker MR, et al. Gender-based violence against adolescent and young adult women in low- and middle-income countries. J Adolesc Health. 2015; 56(2):188-96. 
36. Ahinkorah BO, Dickson KS, Seidu AA. Women decision-making capacity and intimate partner violence among women in sub-Saharan Africa. Arch Public Health. 2018;76:10

37. Beyene AS, Chojenta C, Roba HS, et al. Gender-based violence among female youths in educational institutions of Sub-Saharan Africa: a systematic review and meta-analysis. Syst Rev. 2019;8:59. https://doi.org/10.1186/ s13643-019-0969-9.

38. Christina C. Pallitto, et al., prevalence of intimate partner violence: findings from the WHO multi-country study on women's health and domestic violence. Lancet. 2006;368(9543):1260-9.

39. Uthman OA, Moradi T, Lawoko S. Are individual and community acceptance and witnessing of intimate partner violence related to its occurrence? Multilevel structural equation model. PLoS One. 2011;6(12):8.

40. Abrahams $\mathrm{N}$, et al. Intimate partner violence: prevalence and risk factors for men in Cape Town, South Africa. Violence Vict. 2006;21(p):247-64.

41. Hyman I, Forte T, Du Mont J, et al. Help-seeking rates for intimate partner violence (IPV) among Canadian immigrant women. Health Care Women Int. 2006;27(8):682-94.

42. Djikanovic B, et al. Help-seeking behaviour of Serbian women who experienced intimate partner violence. Fam Pract. 2012;29:189-95.

43. Koenig $M$, et al. Women's status and domestic violence in rural Bangladesh: individual and community-level effects. Demography. 2003;40(2):269-88.

44. Fawole O, Dagunduro AT. Prevalence and predictors of violence against female sex workers in Abuija, Nlgeria. Afr Health Sci. 2014;14(2):215.

45. Fawole OL, Salawu TA, Olarinmoye EOA. Intimate partner violence: prevalence and Pereceptions of married men in Ibadan, Nigeria. Int Q Community Health Educ. 2010;30(4):349-64.

46. Fawole Ol, Asekun-Olarinmoye EO, Osungbade KO. Are very poor women more vulnerable to violence against women?: comparison of experiences of female beggars with homemakers in an urban slum settlement in Ibadan, Nigeria. J Health Care Poor Underserved. 2013;24(4):1460-73.

47. Yaya S, Bishwajit G. Regional prevalence, patterns and correlates of sexual coercion among women in sub-Saharan Africa: a multi-country populationbased study. Int J Sex Health. 2018;30(2):224-35.

48. Uthman OA, Lawoko S, Moradi, T. Factors associated with attitudes towards intimate partner violence against women: a comparative analysis of 17 subSaharan countries. BMC Int Health Hum Rights. 2009;9:14. https://doi.org/10. 1186/1472-698X-9-14.

49. Marteau TM, Hollands GJ, Fletcher PC. Changing human behavior to prevent disease: the importance of targeting automatic processes. Science. 2012;337:1492-4.

50. Gust DA, et al. Factors associated with physical violence by a sexual partner among girls and women in rural Kenya. J Glob Health. 2017;7(2):125-35.

51. Myhill A. Measuring coercive control: what can we learn from National Population Surveys? Violence Against Women. 2015;21(3):355-75.

52. Heise LL. Determinants of partner violence in low and middle-income countries: exploring variation in individual and population-level risk. London School of Hygiene \& Tropical Medicine. 2012. https://doi.org/10.17037/PUBS. 00682451.

53. Barnett R. University knowledge in an age of supercomplexity. High Educ. 2000;40(4):409-22.

54. Zegenhagen S, Ranganathan M, Buller AM. Household decision-making and its association with intimate partner violence: Examining differences in men's and women's perceptions in Uganda. SSM Popul Health. 2019;8: 100442. https://doi.org/10.1016/j.ssmph.2019.100442.

55. Duffy GG, Hoffman JV. In pursuit of an illusion: the flawed search for a perfect method. Read Teach. 1999;53(1):10-6.

56. Kishor S, Johnson K. Domestic violence in nine developing countries: a comparative study. Calverton, Maryland: Macro International; 2004.

57. Yount KM, Carrera JS. Domestic violence against married women in Cambodia. Soc Forces. 2006;85(1):355-87.

58. Johnson MP. Conflict and control: gender symmetry and asymmetry in domestic violence. Violence Against Women. 2006:12(11):1003-18.

59. Porrúa-García C, et al. Development and validation of the scale of psychological abuse in intimate partner violence (EAPA-P). Psicothema. 2016;28(2):214-21.

60. Pico-Alfonso MA. Psychological intimate partner violence: the major predictor of posttraumatic stress disorder in abused women. Neurosci Biobehav Rev. 2005;29(1):181-93.

61. Felson RB. Aggression as impression management. Soc Psychol. 1978:205-13.
62. Graham K, et al. Apparent motives for aggression in the social context of the bar. Psychol Violence. 2013;3(3):218.

63. Kim J, Gray KA. Leave or stay? Battered women's decision after intimate partner violence. J Interpers Violence. 2008;23(10):1465-82.

64. Cattaneo LB, DeLoveh HL. The role of socioeconomic status in helpseeking from hotlines, shelters, and police among a national sample of women experiencing intimate partner violence. Am J Orthopsychiatry. 2008;78(4): 413-22.

65. Miller SL. The paradox of women arrested for domestic violence: criminal justice professionals and service providers respond. Violence Against Women. 2001;7(12):1339-76.

\section{Publisher's Note}

Springer Nature remains neutral with regard to jurisdictional claims in published maps and institutional affiliations.
Ready to submit your research? Choose BMC and benefit from:

- fast, convenient online submission

- thorough peer review by experienced researchers in your field

- rapid publication on acceptance

- support for research data, including large and complex data types

- gold Open Access which fosters wider collaboration and increased citations

- maximum visibility for your research: over $100 \mathrm{M}$ website views per year

At $\mathrm{BMC}$, research is always in progress.

Learn more biomedcentral.com/submissions 